Al Razionalismo, alla sua fede nella verità e nella perfezione, Oakeshott contrappone una visione scettica della conoscenza, che fa da fondamento a una concezione liberale dello Stato nella quale hanno un ruolo centrale le nozioni di 'tradizione' e di 'governo della legge' (Rule of Law). Egli fa propria la visione che si ritrova nell'Illuminismo scozzese e nel fondatore della Scuola Austriaca dell'economia, Carl Menger, ripresa in quegli stessi anni da Hayek, secondo cui lo Stato moderno e le istituzioni politiche sono il frutto di innumerevoli scelte umane, fatte in un lungo arco di tempo, ma non di uno specifico disegno umano. Quelle scelte non erano la risposta ad astratte credenze bensì a specifiche situazioni contingenti, si inserivano all'interno di una specifica tradizione di comportamento. L'idea che vi sia un 'architetto' alla loro base, qualcuno che si sia posto un obiettivo e si sia dedicato a quello scopo specifico, è una fallacia razionalistica. Nella realtà, la tradizione politica di una società fornisce delle 'indicazioni' (intimations) su come eliminare incoerenze al suo interno, per esempio dando il diritto di voto alle donne quando sia apparso evidente che esse hanno la stessa capacità politica degli uomini. Da ultimo, alla visione del Razionalista, che considera il governo un enorme serbatoio di potere che deve essere utilizzato per imporre agli altri la propria visione di una società perfetta, Oakeshott contrappone la visione del 'governo della legge', del rispetto di regole di condotta che consentono a ogni individuo di perseguire la propria personale immagine della felicità. Perché la ricerca della felicità è lo scopo della vita umana.

\title{
OAKESHOTT E HAYEK
}

di Alberto Mingardi*

Lo stesso titolo di questa silloge di Oakeshott, Razionalismo in politica, evoca la critica di Hayek al 'costruttivismo'. Il tema delle convergenze e differenze fra Hayek e Oakeshott è ormai un campo molto arato ${ }^{19}$. Secondo Kenneth Minogue, Hayek e Oakeshott erano "buoni

* Università IULM di Milano.

${ }^{19} \mathrm{Si}$ vedano, fra gli altri, S. COTELLESSA, Il ragionevole disaccordo: Hayek, Oakeshott 
amici"'20. A chi legga le opere dell'uno e quelle dell'altro non sfugge che il punto di partenza era assai diverso: la denuncia della "presunzione fatale" degli ingegneri sociali viene a Hayek dall'aver partecipato al dibattito sul calcolo economico nell'economia di piano, negli anni Trenta. Il liberalismo hayekiano, che promuove il governo limitato in ragione dei limiti cognitivi dei governanti, matura a partire dalla constatazione della hybris dei pianificatori e poi dell'insoddisfazione degli strumenti concettuali degli economisti neoclassici. Oakeshott al contrario è un filosofo idealista e, come scrive Giorgini, è proprio dall'idealismo che matura "una visione del compito della filosofia che informa tutte le sue opere: la filosofia è il proprio tempo appreso nel pensiero; essa deve descrivere e spiegare, non prescrivere"21. La visione dei limiti della filosofia si accompagna a una comprensione del peculiare ambito di ciascuna attività umana, per cui la politica è a sua volta una impresa rigorosamente limitata.

Pur partendo da coordinate intellettuali molto diverse, Hayek e Oakeshott sono stati però esposti alle medesime circostanze storiche. L'idea che l'economia e, dunque, la vita sociale fossero pianificabili in tempo di pace come lo erano state in tempo di guerra, subordinando i fini individuali a un obiettivo collettivo (imporsi sulla Germania o garantire a tutti un certo stock di 'diritti sociali') è il leit motif che mette in moto la loro riflessione nell'immediato dopoguerra. Ciò è particolarmente evidente nel caso di Oakeshott: Razionalismo in politica, il saggio che dà il titolo al volume, è infatti del 1947, di due anni successivo alla vittoria elettorale dei laburisti e precede di un anno la nascita ufficiale, il 5 giugno 1948, del National Health Service.

Coloro che insistono sulla distanza fra Oakeshott e Hayek tendono

e le regole immotivate della società, Milano, Vita e Pensiero,1999; L. MARSH, Situating the Mind: Hayek and Oakeshott, in P. Franco, L. Marsh (eds.), A Companion to Michael Oakeshott, Philaldephia, Pennsylvania University Press, 2012, pp. 248-267; C.-Y. Cheung, The Critique of Rationalism and the Defense of Individuality: Oakeshott and Hayek, in "Cosmos+Taxis. Studies in Emergent Order and Organization", n. 3, 2014, pp. 3-9; G. Callahan, Hayek and Oakeshott on Rationalism, in "Voegelin Review", 26 giugno 2017, https://voegelinview.com/hayek-oakeshott-rationalism/

${ }^{20}$ Cfr. K. Minogue, The Elusive Michael Oakeshott, in "The American Conservative", 1 ottobre 2009, https://www.theamericanconservative.com/articles/the-elusive-oakeshott/

${ }^{21}$ G. Giorgini, Presentazione al pubblico italiano, in M. OAKESHOTt, Razionalismo in politica, cit., p. 9. 
a ricordare come il primo liquidasse con queste parole La via della schiavitù: "un piano per resistere a tutte le pianificazioni può essere meglio del suo contrario, ma appartiene allo stesso stile di fare politica” (p. 74). Il rifiuto 'ideologico' dell'intervento pubblico sarebbe la spia di "una società già profondamente infettata dal Razionalismo", che trasforma in "un'impacciata ideologia" quelle che erano "risorse tradizionali di resistenza alla tirannia del Razionalismo" (p. 74). Se si considera il saggio che, nel 1976, una discepola intellettuale di Oakeshott, Shirley Robin Letwin dedica ad Hayek, si comprende però che Oakeshott tutto vuol fare tranne che un elogio del pragmatismo economico che consenta di dare un colpo al cerchio e uno alla botte, che mette assieme un po' di economia di mercato e un po' di prestidigitazione macro-economica. L'esatto contrario. La ragione della diffidenza sta precisamente nel fatto che Hayek si ostina a discutere di temi quali "l'istruzione dei bambini, le regolazioni urbanistiche, i sussidi agli agricoltori" 22 cioè giustificare una alternativa "liberista" sul medesimo terreno di gioco degli interventisti, fornendo prove per la maggiore efficienza di un approccio "di mercato" nel rispondere a determinate esigenze. Del resto, Oakeshott era tutto fuorché indifferente alla libertà economica: la libertà di commercio con l'estero era per lui "una delle tutele più preziose e più efficaci che una comunità possa avere contro il potere eccessivo" (p. 198), e la "proprietà privata di altre risorse oltre alla capacità personale" (p. 192) era essenziale alla libertà.

Hayek cita Oakeshott rispetto all'uso dei termini "teleocratico" e "nomocratico" in Legge, legislazione e libertà23, e, prima, sull'America in The Constitution of Liberty, del 1960. Se per Oakeshott la Rivoluzione americana e, in particolare, la Dichiarazione d'Indipendenza sono "un caratteristico prodotto del saeculum rationalisticum" (p. 84), Hayek in qualche modo difende l'esperienza americana: "tutte le nuove scoperte della Costituzione [...] erano dovute o all'applicazione di principi

22 S. Robin Letwin, The Achievement of Friedrich A. Hayek, in F. Machlup (ed. ), Essays on Hayek, Hillsdale, Hillsdale College Press, 1976, p. 147. Questo catalogo di temi, nel testo della Letwin, si riferisce all'opera hayekiana The Constitution of Liberty, London, Routledge, 1960.

${ }^{23}$ F.A. HAYeK, Law, Legislation and Liberty, 3 vols., London, Routledge, 1973-79; trad. it Legge, legislazione e libertà, Milano, il Saggiatore, 1986, p. 200. 
tradizionali a problemi particolari o erano emerse come conseguenze di idee generali solo indistintamente percepite"24.

In realtà, le circostanze accompagnarono le riflessioni di Oakeshott e Hayek in direzioni molto simili. Per entrambi la riflessione sulla politica è inscindibile da una teoria della conoscenza. Per entrambi la 'presunzione fatale' è in buona sostanza una deduzione erronea, circa i gruppi umani, dal successo che abbiamo ottenuto nell'intervenire sulla natura. Per Oakeshott il 'mito della politica razionalista' è l'“assimilazione della politica all'ingegneria" (p. 55); per Hayek il 'costruttivismo' è l'applicazione del "tecnicismo ingegneristico alla soluzione dei problemi sociali"25. Entrambi considerano la Rule of Law "la concezione di Stato più civilizzata e meno ingombrante sino ad ora messa a punto" 26 e ne rintracciano la fonte nell'esperienza storica, non nell' elaborazione teorica. Forse la differenza cruciale fra i due è data dal fatto che Hayek si adopera per "adattare" la rule of law per fornire una risposta liberale ai problemi del Novecento mentre Oakeshott non si pone il problema.

\section{LE MASSE E LA DEMOCRAZIA RAPPRESENTATIVA}

di Giovanni Orsina*

In Razionalismo in politica il saggio Le masse nella democrazia rappresentativa occupa poche pagine. Ma le dimensioni ingannano, e molto: in uno spazio angusto, con una forza e una lucidità davvero notevoli, Oakeshott condensa una storia dell'individuo e dei suoi nemici dal tardo Medioevo al ventesimo secolo, e la costella di dettagli illu-

${ }^{24}$ F.A. HAYEK, The Constitution of Liberty, cit.; trad. it. La società libera, Soveria Mannello, Rubbettino, 2007, p. 394.

${ }^{25}$ F.A. HAYEK, The Counter-Revolution of Science: Studies on the Abuse of Reason, Glencoe, Ill., The Free Press, 1952; trad. it. L'abuso della ragione, Soveria Mannelli, Rubbettino, 2008, p. 164.

26 M. OAKEShott, The rule of law, in M. OAKeshott, On History and Other Essays, del 1983, ed. by T. FULLER, Indianapolis, LibertyFund, 1999, p. 178.

* Luiss School of Government. 
minanti. Ora, un saggio così ricco può essere affrontato in molti modi. Se ne potrebbero rintracciare gli ascendenti intellettuali, ad esempio. Lo si potrebbe collocare nel dibattito della sua epoca, visto che in quegli anni di quei temi si occupavano in tanti, e non dei meno brillanti. $\mathrm{O}$ potrebbero definirsene il posto e il ruolo nella produzione dell'autore. Qui non farò nulla di tutto questo, sia perché lo ha già fatto Giorgini nell'introduzione al volume, sia - e soprattutto - perché non ne ho le competenze. Mi concentrerò invece sul nucleo centrale dell'argomentazione del saggio e sul valore notevole che può avere ancora oggi, a sessant'anni dalla sua prima pubblicazione.

Come altri scritti di Oakeshott, anche questo si regge su una dicotomia, in questo caso fra individuo e anti-individuo. L'opzione di valore del filosofo inglese non potrebbe essere più esplicita fin dall'inizio dello scritto, che a buon bisogno, nel caso in cui il lettore particolarmente ottuso o distratto non l'avesse a quel punto ancora capito, si conclude con questa frase: "sotto ogni aspetto importante l'individuo appare ancora la sostanza e 1' 'anti-individuo' solamente l'ombra" (p. 180). Nel corso dell'analisi, però, questo schema apparentemente elementare mostra tutta la propria complessità, e anche la separazione fra le due categorie perde parte della propria nettezza se non teorica, quanto meno empirica.

L'individuo moderno - afferma Oakeshott, debitore qui della lezione di Jacob Burckhardt - nasce in Italia alla fine del XIII secolo e, irradiandosi dalla Penisola, dà avvio a una trasformazione rivoluzionaria in ogni ambito: psicologico, etico, sociale, politico. "Comparve una nuova immagine della natura umana - non Adamo, non Prometeo ma Proteo - un' indole distinta da tutte le altre in base alla propria molteplicità e alla propria infinita capacità di trasformare se stessa" (p. 162). Ma questa rivoluzione, "che alcuni salutarono come felicità, appariva ad altri come sconforto. La medesima condizione dell'esistenza umana fu vista sia come progresso sia come decadenza" (p. 167). Di fronte all'individuo sorgeva così 1' "individuo manqué", destinato in breve tempo a convertirsi senz'altro in anti-individuo militante, nel momento cui si sarebbe reso conto di come, "lungi dall'essere solo, egli apparteneva alla classe più numerosa della società europea moderna, la classe di coloro che non avevano scelte proprie da fare" (p. 169). Allo stesso modo dell'individuo, anche l'anti-individuo avrebbe dato vita a una propria morale e a un proprio tipo di governo, il governo po- 
polare, apparentemente simile ma in realtà del tutto differente da quello parlamentare generato dall'individualismo.

La dicotomia, prosegue Oakeshott, non ha origini né economiche né intellettuali, ma etiche. L'anti-individuo, che il filosofo inglese pur polemizzando implicitamente con José Ortega y Gasset chiama anche uomo-massa, "non è necessariamente 'povero', né è invidioso soltanto delle 'ricchezze'; non è necessariamente 'ignorante', spesso è un membro della cosiddetta intelligentsia; appartiene a una classe che non ha eguali. È caratterizzato fondamentalmente da un'inadeguatezza morale, non intellettuale". E tuttavia, fra quanti ci circondano nel mondo reale non possiamo distinguere con troppa chiarezza i 'buoni' dai 'cattivi' nemmeno su basi etiche, poiché "ogni essere umano europeo ha una propensione verso questa attitudine a essere un 'anti-individuo'; 1' 'uomo massa' è semplicemente quello in cui questa propensione è dominante" (pp. 177-178). Abbiamo già visto, poi, come quella degli anti-individui sia la classe più numerosa. Questa loro forza quantitativa si combina però con un'indiscutibile inferiorità qualitativa. Il vigore della morale individualistica, infatti, è stato tale che "non soltanto spazzò via i resti della morale appropriata al defunto ordine comunitario, ma lasciò anche poco spazio per un'alternativa a se stessa" (p. 168). A tal punto che, quando l'anti-individualismo è riuscito infine a costruirsi una propria morale, ha generato, "sotto molti aspetti, una creatura rachitica: essa non raggiunse mai una forma paragonabile a quella che Hobbes, Kant o Hegel diedero alla morale dell'individualità; essa inoltre non è mai stata in grado di resistere alla ricaduta nei concetti a lei inappropriati dell'individualità' (p. 173).

Proprio a dimostrazione della sua forza inarrestabile, nei sessant'anni che ci separano dal momento in cui questo saggio è stato scritto l'etica dell'individualismo si è affermata come mai prima. Accompagnata però da un'ondata di ottimismo semplicistico, soprattutto fra gli anni Ottanta e i Novanta, dalla quale forse, se avessimo letto Oakeshott con maggiore attenzione, non ci saremmo fatti trascinare così facilmente. L'anti-individualismo non sa proporre alternative storicamente vitali, ma è presente in ciascuno di noi ed è forte in segmenti molto ampi, spesso maggioritari, della popolazione. Poiché la sua scaturigine è etica, poi, né il benessere materiale né l'istruzione riescono ad attenuarne più di tanto l'impatto. Proteo è più che mai il dio della tarda modernità, ma gli esseri umani appaiono più che mai a disagio nel suo 
regno proteiforme: "non [...] possono star fermi. Corrono via dalle loro stesse metamorfosi. Non rimangono ad assistervi, se le anticipano, preferiscono essere tutto, tranne quello che potrebbero", scriveva Elias Canetti già nel 194227. Ci sarebbe anzi da chiedersi, seguendo lo spunto che Oakeshott soltanto accenna nell'ultima citazione riportata sopra, se e fino a che punto l'operazione in virtù della quale l'anti-individualismo si è da ultimo appropriato della morale individualistica per rovesciarla in uno strumento di conformismo, disciplinamento e omogeneizzazione, non rappresenti in realtà un estremo tentativo di mediazione - precario, contraddittorio e illiberale - fra l'inevitabilità di Proteo e la sua impossibilità.

\begin{abstract}
Michael Oakeshott (19011990) was one of the great philosophers of 20th century England. Yet his name is seldom known in Italy and his influence in Italian circles was modest. This section of 'Il Politico' features a few essays reflecting on Oakeshott in the occasione of the translation of Rationalism in Politics by Giovanni Giorgini. The contributors to this section endeavour to present different facets of the larger picture of Oakeshott's political thought, emphasizing its many different nuances.

Sergio Belardinelli reflects on the Tower of Babel in Oakeshott's thinking. He finds in Oakeshott "an invitation to remain in the fragmentation of the world in which we live, rather than attempting to move over it".

Agostino Carrino focuses on Oakeshott's bonum civile and the foundations of political society, taking Hegel and Carl Schmitt as points of reference. Thinking of Oakeshott in the wider framework of the history of political thought is necessary, for an author who devoted so much attention to the history of
\end{abstract}

ideas, being, among other things, the editor of a famous edition of Leviathan.

Hence Raimondo Cubeddu interprets Oakeshott in the light of the latter's rejection of Utilitarianism and of his blunt criticism of Jeremy Bentham, who, in Oakeshott's opinion, never had a genuine interest for speculative thinking.

Giovanni Giorgini places Oakeshott's reflection on rationalism in politics in the history of contemporary political thought, on a par with other criticism of social engineering such as F.A. Hayek's and Karl Popper's, and points out the difference between Oakeshott and such authors in his understanding of the rule of law and the nature of society.

Alberto Mingardi presents a quick comparison between Oakeshott and F.A. Hayek, highlighting common elements rather than the differences between the two.

Giovanni Orsina looks at Oakeshott's work, thinking of the development of mass politics and the role played by individualism in modern societies.

27 Cfr. E. CAnetti, Die Provinz des Menschen, München, Hanser, 1973; trad. it. La provincia dell'uomo, Milano, Adelphi, 1978, p. 24. 